\title{
Enantiomeric Structures of a Self-Assembling 3D DNA Crystal Scaffold
}

\author{
Chad R. Simmons, Fei Zhang, Tara MacCulloch, Nour Eddine Fahmi, Nicholas Stephanopoulos, \\ Yan Liu \& Hao Yan \\ Arizona State University
}

Structural DNA nanotechnology uses DNA as a molecular building block to create programmable self-assembled structures. One of the most promising applications for this technology is the design of lattices that can serve as artificial scaffolds to organize guest molecules in three dimensions. In previous work, we designed self-assembling DNA crystal constructs in order to place macromolecules of interest-such as peptides, proteins, RNA, or viruses - onto DNA scaffolds. In this work, we have determined the crystal structures of mirrorimage isomers of DNA lattices. DNA appears naturally with a right-handed helical twist (DDNA), but we hypothesized that using the L-enantiomer (L-DNA) would result in motifs that could assemble into either crystal hand. L-DNA duplexes possess identical physical attributes as the corresponding D-DNA, except for its left-handed double helix. Here, we explore both enantiomers of a motif that crystallizes into a matrix of layers organized by a series of Holliday junctions, resulting in a 3D lattice. Each layer is linked by an oligonucleotide containing 4 repeats of a 6-base sequence. Bromine derivatized crystals were used for phase determination of the L-DNA structure by incorporation of the heavy atom on 4 thymine bases in each asymmetric unit. In parallel, we prepared selenium derivatives of the D-DNA motif via the organic synthesis of a seleno-dU phosphoramidite for incorporation into the right-handed crystal system. Phases were acquired for both forms via SAD, and the resulting structures were determined to $3.0 \AA$ resolution. The right- and left-handed forms crystallized in the trigonal space group $P 3$ with mirror image three-fold helical screw axes $P 3_{2}$ and $P 3_{1}$ for each motif, respectively. The structures reveal an organized, periodic array of discretely defined cavities, well suited to host guest molecules and potentially allow us to dictate a priori the assembly of guest-DNA conjugates with a specified crystalline hand. Furthermore, D-DNA is highly susceptible to digestion by DNases, whereas L-DNA is completely resistant, making it an attractive choice as a protective scaffold for guest molecules. Crystals for each isomer were obtained in identical buffer conditions, incubated in the presence of DNase, and monitored for stability over time. Degradation of the D-DNA crystals was clearly observable within 4 hours, with complete digestion occurring within 8 hours. By contrast the L-enantiomeric crystals did not demonstrate any degradation over multiple days, indicating their complete resistance to DNases. Our L-DNA structure is entirely novel, and allows for the design of periodic arrays of macromolecules that can maintain stability without compromise from nucleases. The structures of these selfassembling mirror image DNA crystal systems provide an exciting new route towards the sitespecific incorporation of guest molecules within 3D lattices for DNA directed crystallization. 